\title{
Time after Time: Representing Time in Literary Texts
}

\author{
Michael Levison \\ School of Computing \\ Queen's University, Canada \\ levisonecs.queensu.ca
}

\author{
Greg Lessard \\ French Studies \\ Queen's University, Canada \\ greg.lessard@queensu.ca
}

\begin{abstract}
The representation of temporal information in text represents a significant computational challenge. The problem is particularly acute in the case of literary texts, which tend to be massively underspecified, often relying on a network of semantic relations to establish times and timings. This paper shows how a model based on threaded directed acyclic graphs makes it possible to capture a range of subtle temporal information in this type of text and argues for an onomasiological approach which places meaning before form.
\end{abstract}

\section{Time and Text}

This paper deals with the representation of temporal phenomena in literary texts. As a result, it builds on work from a number of fields. ${ }^{1}$ Thus, it takes as given the longstanding observation from philosophy that time is not a simple issue of days, months and years, but reflects issues of perception and culture (Ricoeur, 1983). At the same time, it assumes that the study of temporal phenomena will be enhanced by use of a formal representation (Allen, 1984). It further assumes the traditional narratological distinction between the information which underlies a text, variously known as the $f a b$ ula or histoire and which we will henceforth call the story and some particular instantiation of this in text, often called the sjuzhet or récit, which we will henceforth call the narrative (Genette, 1972).

Mani (2010), based on earlier work, suggests that the temporal relations BEFORE, DURING, IMMEDIATELY BEFORE, BEGINS, ENDS, SIMULTANEOUS AND OVERLAPS are adequate for representing time in human languages. This raises the interesting empirical question of how

\footnotetext{
${ }^{1}$ In what follows, for lack of space, we restrict ourselves to citing some typical examples from a vast literature.
}

well this model applies to literary texts, given their complex but typically underspecified nature. In fact, in the case of time, a literary text often gives no explicit indication of temporal phenomena, but relies on encyclopedic knowledge available to the reader. In addition, we might ask how different temporal relations are distributed across literary texts, as compared with what is found in expository or other types of texts, or simpler narratives. At the same time, as Lascarides and Asher (1991) point out, it is important to see temporal relations as a subset of a broader range of relations including Narration, Explanation, Background, and Result, all of which have temporal implications.

There does exist a growing body of analyses of narrative texts, but most of these are based on relatively simple third person narratives such as fables. Such texts tend to be event-driven (one thing follows another) and they tend to lack more complex literary phenomena such as first person narrative, where all information is not known, multiple, sometimes competing, perspectives, and significant temporal shifts. It will be important to analyse literary texts in their full complexity before we are capable of pronouncing on the use of time. This will no doubt be aided by research on narrative generation, such as (Callaway and Lester, 2002), (Riedl and Young, 2012), and (Montfort, 2007), where temporal representations at the abstract level are made use of, but this must be complemented by empirical work on actual texts.

The empirical study of temporal relations in complex literary texts will be complicated by the fact that, despite recent progress (for example, Kolomiyets et al. (2012)), parsers still do not match the performance of humans in assigning temporal points and relations. As a result, building a detailed corpus of literary texts will still take some time and much human effort. When it is undertaken, one of the fundamental decisions to be faced will be what is to be represented. Most 
work to date takes texts themselves as the object of tagging, and schemes such as TimeML (Pustejovsky et al., 2003) are designed to allow quite precise temporal information to be added to texts in the form of markup. As a result, they focus on phenomena in the narrative rather than in the story. To put this another way, they adopt a semasiological perspective (from form to meaning), rather than an onomasiological one (from meaning to form) (Baldinger, 1964). However, it is reasonable to ask whether the appropriate level of representation should in fact be one level up, at the level of the story. We argue here that this is the case. Elson (2012) takes a first step in this direction by mapping the textual layer to a propositional layer; however, most of the texts he deals with are relatively simple. We will show below how, in some complex examples, representing temporal phenomena at the story level requires at the least additional 'machinery' based on multiple points of view, temporal shifts including prolepsis, and encyclopedic knowledge, but that it also offers insights into narrative structure not apparent at the textual level.

\section{DAGs and Threading}

The story which underlies a literary text may be represented by means of a directed acyclic graph, henceforth DAG, composed of nodes connected by unidirectional edges. The acyclicity requires that no sequence of edges may return to an earlier node. The nodes carry segments of meaning represented by semantic expressions. These are functional representations, described in (Levison et al., 2012). Each semantic expression is composed of elements drawn from a semantic lexicon.

A simple example might be stab (brutus, caesar), where the two entities brutus and caesar denote certain human beings, and stab is a function which takes two entities and returns a completion $^{2}$ having the obvious meaning. On the basis of functions such as these, it is possible to construct the DAG shown in Fig. 1. ${ }^{3}$

The unidirectional edges between the various

\footnotetext{
${ }^{2}$ A completion may be thought of as the semantic equivalent of an utterance, an entity as the semantic equivalent of a noun, and an action as the semantic equivalent of a verb.

${ }^{3}$ The DAGs shown here were constructed with yEd (http: //www.yworks.com/en/products_yed_ about.html), which generates a GraphML representation for each graph. For simplicity, we have ignored representation of tense and aspect in these examples, although the formalism permits this.
}

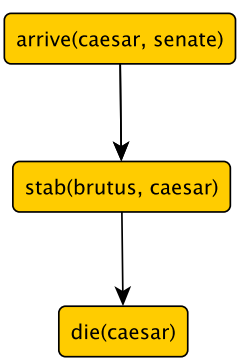

Figure 1: A DAG for the various states of Caesar

nodes represent semantic dependency, that is, the fact that subsequent nodes depend upon information contained in previous nodes, and by transitive closure, parents of previous nodes. So, in Fig. 1, the expression stab (brutus, caesar) depends on the fact that Caesar is at the Senate, while Caesar being dead depends on the stabbing. The relation carried by edges may be one of order (one node occurs before another), or of some sort of causality, whereby a subsequent node is made possible by a previous node. In addition, nodes which convey a coarse level of meaning may themselves be refined into DAGs at a finer level. And so on, recursively.

Since a DAG exists prior to any text which represents it, a text may begin at the start of the DAG and follow the nodes, as in Caesar arrived at the Senate, then Brutus stabbed him, then he died, or alternatively at the end, as in Caesar died because Brutus stabbed him after his arrival at the Senate, in the middle, as in Brutus stabbed Caesar after he arrived at the Senate, and then he died, or even in a zigzag order, as in Caesar arrived at the Senate and then died because Brutus stabbed him. ${ }^{4}$

Each of these narrations may be represented by a sequence of nodes, in other words, a thread, showing the order in which the meaning carried by the nodes is presented to the reader. Note that the thread passes through some or all of the nodes, but need not follow the edges of the DAG. Nor is it constrained to be acyclic: it may visit the same node more than once. An example of this is provided by a narration in which the same event is recounted twice. To take an extreme case, in the movie Groundhog Day (http://www.imdb. com/title/tt0107048/), the character Phil relives the same day and its events many times.

In our DAGs, we represent threads by a dot-

\footnotetext{
${ }^{4}$ For more examples, see (Mani, 2012).
} 
ted line to distinguish them from the edges of the DAG. By threading coarse or refined DAGs, the narration can be at different levels of detail. In addition, a single DAG may be traversed by multiple threads representing, among other things, different points of view. So, for example, suppose that a third party, say Livia, finds Caesar's dead body, observes the stab wounds, and concludes that a previously living Caesar has been killed. From the point of view of the Livia thread, the 'Caesar is dead' node is traversed before the stabbing node (although from Livia's point of view, it may not be clear who has done the stabbing). Alternatively, a fourth character may observe a stabbing in the distance, then on approach note that the stabbee is Caesar, assumed alive until that point, and finally learn that Caesar is dead.

\section{Relative and Absolute Timestamps}

Within the DAG model, the simple chronological ordering of events or activities requires no extra features except perhaps 'colouring' certain edges to distinguish between those which denote chronological dependence and those whose dependence is based on other reasons. Figure 1 above illustrates this. However, more complex temporal relationships such as 'while' can be signified by nodes indicating relative or absolute times, as in:

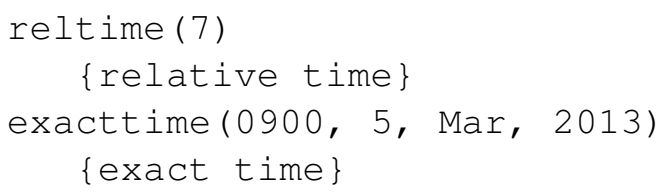

Consider, for example, the DAG shown in Fig. 2 . Here, both event 1 and event 2 take place after reltime (7) and before reltime (8)..$^{5}$ If no other activities take place in the same context, we might conclude that while event 1 was taking place, event 2 was happening elsewhere. Both events conclude before event4. In addition, event 3 occurs after event 1 , but it may have started before or after reltime (8); and there is no information about its relation to event 4 . Additional arguments can be added to specify whether an event is durative or punctual, because nothing says that event 1 actually began at reltime(7) and ended at reltime(8). The function exacttime () allows us to anchor parts of the DAG at, or more precisely after, specific moments.

\footnotetext{
${ }^{5}$ The parameters to reltime, by the way, are irrelevant; they are included only for the purposes of this commentary.
}

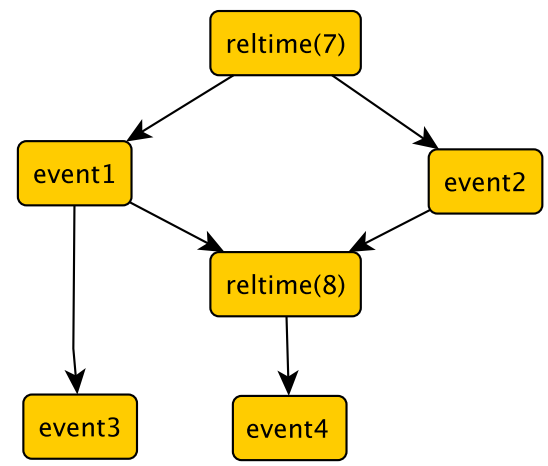

Figure 2: A DAG showing relative times and events

\section{Some Empirical Tests of the Formalism}

To empirically test the model proposed here, we will examine several actual texts. Of course, these represent only a small selection of a vast range of temporal phenomena. Our object is simply to show how the proposed model may be applied.

\subsection{Prolepsis}

As noted earlier, a literary text may bring into play a variety of perspectives. One of these is prolepsis, or foreknowledge of a future event. Consider the following example from Homer's Iliad. ${ }^{6}$ Achilles asks Zeus for success in a battle and that Patroclus survive the battle. Zeus grants the first wish, but not the second. ${ }^{7}$ As a result, he (Zeus) and by extension, we, as readers, know that $\mathrm{Pa}$ troclus will die. However Patroclus himself is unaware of this. We may represent this part of the story by means of the DAG shown in Fig. 3, which contains two sets of dependencies, one which links Zeus to the decision that Patroclus will die, and the other which links Patroclus to his fighting and dying. We may then capture the temporality of the narrative by threading this DAG. ${ }^{8}$

An example like this may seem arcane, but cases of multiple points of view, or multiple threading, are found in a variety of textual models. Thus, in a murder mystery, the detective comes to understand the ordering of particular events, including the murder, and may subsequently explain

\footnotetext{
${ }^{6}$ This instance of prolepsis is discussed in Grethlein (2010).

${ }^{7}$ Iliad, ch. 16, v. 249, http://classics.mit. edu/Homer/iliad.html.

${ }^{8}$ Threads are shown here by numbered dotted lines to indicate their order, while dependencies are shown by unnumbered solid lines.
} 


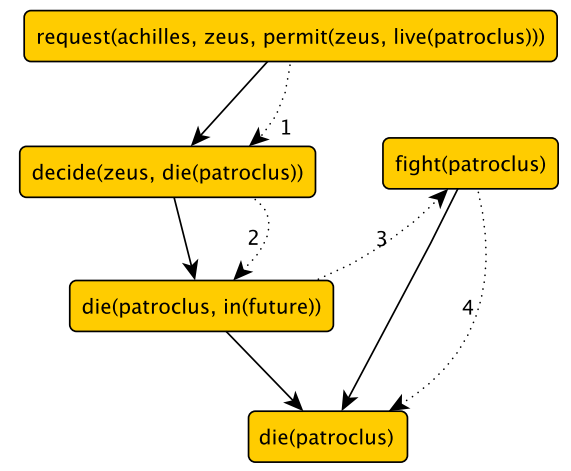

Figure 3: A DAG for part of the Iliad

this to the reader.

\subsection{Parallel Series of Events}

Consider the following passage from the Conan Doyle story entitled The Red-headed League. ${ }^{9}$

When I saw him that afternoon so enwrapped in the music at St. James's Hall ... ${ }^{10}$

"You want to go home, no doubt, Doctor," he remarked as we emerged.

"Yes, it would be as well."

"And I have some business to do which will take some hours. ... to-day being Saturday rather complicates matters. I shall want your help to-night."

"At what time?"

"Ten will be early enough."

"I shall be at Baker Street at ten."

... It was a quarter-past nine when

I started from home and made my way ... to Baker Street. ... On entering his room I found Holmes in animated conversation with two men, ...

The text itself provides two absolute times, one prescriptive, that of the time when Watson is to meet Holmes, and the other descriptive, the time reported by Watson for his leaving home. Another more approximate time is also provided, the fact that Watson and Holmes are listening to music in St James's Hall on a Saturday afternoon. All of these could be marked up in the text itself. However, others would provide a greater challenge. On Watson's return to meet Holmes, he discovers that

\footnotetext{
${ }^{9}$ First published in the Strand magazine in 1891. See http: //www. gutenberg.org/ebooks/1661.

${ }^{10}$ Several non-pertinent elements of the text have been elided. These are shown by suspension points.
}

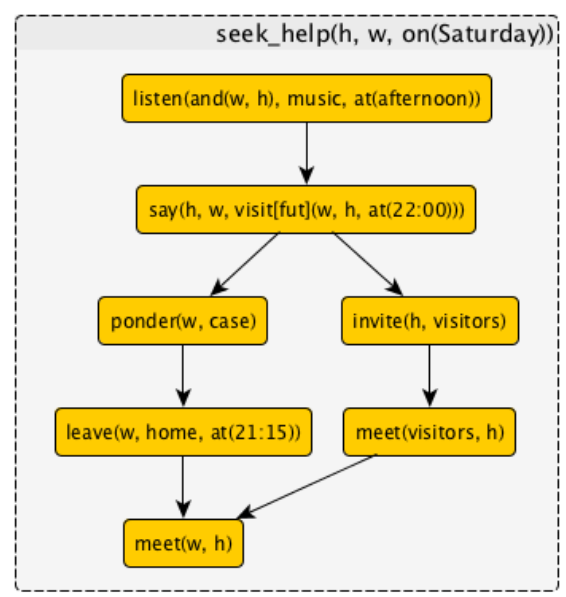

Figure 4: A DAG for part of the Red-headed League

others are present, presumably at Holmes' invitation, although this is not specified in the text itself. The chronology of Watson's activities is provided only by its placement in the text, between the conversation with Holmes and the return to meet Holmes, while the arrival of the others cannot be marked up at all at the textual level since it is not even mentioned in the text. Such a model provides a serious challenge to a semasiological markup, for obvious reasons. However, it may be easily represented by a DAG, as shown in Fig. 4 .

Note that the nodes of the DAG are all enclosed in a higher-level node situated on Saturday. This 'envelope' provides the framework for the detailed events. However, within this envelope, a branching occurs, separating Watson's explicitly noted activities from those which we must suppose Holmes to have accomplished. The two series are bracketed between a relative temporal marker (the moment when Watson and Holmes leave each other) and an absolute temporal marker (Watson's arrival at Holmes' lodgings around 10).

\subsection{Access to Encyclopaedic Information}

Reading a text is not a simple activity. Among other things, it requires a constant reference to background 'encyclopaedic' information. The nature of this information will vary from reader to reader. As an illustration, consider the following paragraph, which opens Flaubert's novel Salammbô. ${ }^{11}$

\footnotetext{
${ }^{11} \mathrm{We}$ provide here the English translation from http://www.gutenberg.org/files/1290/ 1290-h/1290-h.htm\#link2HCH0001.
} 


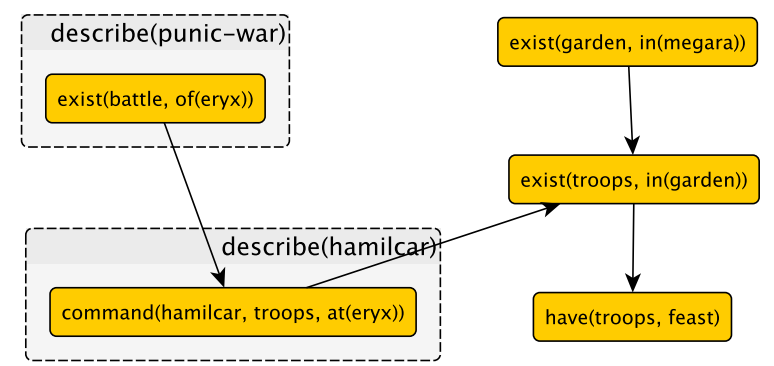

Figure 5: A DAG for the introduction to Salammbô

It was at Megara, a suburb of Carthage, in the gardens of Hamilcar. The soldiers whom he had commanded in Sicily were having a great feast to celebrate the anniversary of the battle of Eryx, and as the master was away, and they were numerous, they ate and drank with perfect freedom.

At the most basic level, any reader may use the tense (had commanded) and some lexical items (anniversary) to determine the anteriority of the battle of Eryx with respect to the feast. However, more educated readers will probably be able to use the proper name Carthage to locate the text in the far past, while even more educated readers will be able to use the names Hamilcar and Eryx to place the feast after the period 244-242 BCE.

We may represent the interplay between what is given by the text and the information available to the reader (which, importantly, is also representable by a DAG) as shown in Fig. 5, where we see that the node exist(troops...), represented in the text, depends on the node command (hamilcar...) also represented in the text. However, this latter node is a subnode of the higher-level node describe (hamilcar), which provides information (including temporal information) not present in the text. Similarly, the node exist (battle...), present in the text, is part of another higher-level node (describe (punic-war)), which contains more detailed encyclopaedic information.

This model captures both the temporal elasticity provided by the interplay of logical dependency and the varying levels of temporal assignment noted above. To put this another way, it captures the set of readings which the same text may carry for different readers. In particular, different readings may thread this DAG at different levels of granularity, some coarse, some finer.

\section{Conclusions and Next Steps}

Although they are limited to issues of time, the examples studied above suggest that an onomasiological approach gives access to textual and literary phenomena which escape tagging of textual contents alone. While the use of DAGs and threading currently requires human intervention, the output of the model, by its formality, provides a means of studying in detail the instantiation of stories as narratives, and thereby, a complement to existing approaches to literary time.

\section{References}

James F. Allen. 1984. Towards a general theory of action and time. Artificial Intelligence, 23:123-154.

Kurt Baldinger. 1964. Sémasiologie et onomasiologie. Revue de linguistique romane, 28:249-272.

Charles Callaway and James Lester. 2002. Narrative prose generation. Artificial Intelligence, $139(2): 213-252$.

David K. Elson. 2012. Modeling Narrative Discourse. $\mathrm{PhD}$ thesis, Columbia University.

Gérard Genette. 1972. Figures III. Éditions du Seuil, Paris.

Jonas Grethlein. 2010. The narrative reconfiguration of time beyond Ricoeur. Poetics Today, 31(2):313329.

Oleksandr Kolomiyets, Steven Bethard and MarieFrance Moens. 2012. Extracting narrative timelines as temporal dependency structures. In Proceedings of the 50th Annual Meeting of the Association for Computational Linguistics (ACL'2012), pp. 88-97.

Alex Lascarides and Nicholas Asher. 1991. Discourse relations and defeasible knowledge. In Proceedings of the 29th Annual Meeting of the Association of Computational Linguistics (ACL91), pp. 55-63.

Michael Levison, Greg Lessard, Craig Thomas, and Matthew Donald. 2012. The Semantic Representation of Natural Language. Bloomsbury Publishing, London.

Inderjeet Mani. 2012. Computational Modeling of Narrative. Morgan and Claypool, San Rafael, CA.

Inderjeet Mani. 2010. The Imagined Moment: Time, Narrative and Computation. University of Nebraska Press, Lincoln, Nebraska. 
Nick Montfort. 2007. Ordering events in interactive fiction narratives. In Proceedings of the AAAI Fall Symposium on Interactive Narrative Technologies. Technical Report FS-07-05, B.S. Magerki and M. Riedl, eds., AAAI Press, Menlo Park, CA, pp. 8794.

James Pustejovsky, Jose M. Castaño, Robert Ingria, Roser Saurí, Robert Gaizauskas, Andrea Setzer, and Graham Katz. 2003. TimeML: Robust specification of event and temporal expressions in text. In Fifth International Workshop on Computational Semantics (IWCS-5).

Paul Ricœur. 1983. Temps et récit. Volume 1. Éditions du Seuil, Paris.

Mark Riedl and R. Michael Young. 2010. Narrative planning: balancing plot and character. Journal of Artificial Intelligence Research, 39:217-268. 